\title{
Note on the Spectrum of Some Schrödinger Operators*
}

\author{
By \\ Teruo Ushijima ${ }^{\dagger}$
}

\section{$\S 0$. Introduction}

Recently S. T. Kuroda has developed a new stationary method of perturbation of continuous spectra using the technique of factorization of the perturbation term ([2], [3]). The object of this note is to show that his theory can be applied to $n$-dimensional Schrödinger operators which have first order differentiations with variable coefficients. Namely we consider the differential operator:

$$
L=\sum_{j=1}^{n}\left(\frac{\partial}{i \partial x_{j}}+b_{j}(x)\right)^{2}+q(x)
$$

where $b_{j}$ and $q$ are real valued functions. The case of $n \geqslant 3$ will be treated in this note. This problem has been already treated by Kuroda $([4])$ in the case of $b_{j}(x)=0$. In this case our work agrees with his result.

The author expresses his hearty thanks to Professor S. T. Kuroda and Professor $T$. Ikebe who kindly read the draft of this note and gave him valuable advices.

\section{$\S 1$. Statement of the Results}

Let $H_{0}$ be the self-adjoint realization of $-\Delta$ in $L^{2}\left(R^{n}\right)$, where domain $\mathfrak{D}\left(H_{0}\right)=\mathscr{D}_{L^{2}}^{2}\left(R^{n}\right)$. We consider the following conditions :

Received July 18, 1968.

Communicated by S. Matsuura.

* This work was partly supported by the Sakkokai Foundation.

$\dagger$ Department of Pure and Applied Sciences, Institute of Mathematics, Faculty of General Education, University of Tokyo. 
(I) For some constants $a, p_{1}$ and $p_{2}$ satisfying

$$
a>\frac{n}{2}, 2 n>p_{1}>n \text { and } 2 n>p_{2}>\max \left(2, \frac{n}{2}\right),
$$

it holds that

$$
\left\{\begin{array}{l}
(1+|x|)^{a} b_{j}(x) \in L^{p_{1}}\left(R^{n}\right) \quad(1 \leqslant j \leqslant n), \\
(1+|x|)^{a} q^{\prime}(x) \in L^{p_{2}}\left(R^{n}\right),
\end{array}\right.
$$

where $b_{j}(x)$ are continuously differentiable functions and

$$
q^{\prime}(x)=\sum_{j=1}^{n}\left(\frac{\partial}{i \partial x_{j}} b_{j}(x)+b_{j}{ }^{2}(x)\right)+q(x) .
$$

(II) For some $p_{3}$ with $n>p_{3}>2$, it holds that

$$
\left\{\begin{array}{l}
(1+|x|)^{a} b_{j}(x) \in L^{p_{3}}\left(R^{n}\right) \quad(1 \leqslant j \leqslant n), \\
(1+|x|)^{a} q^{\prime}(x) \in L^{p_{3}}\left(R^{n}\right) .
\end{array}\right.
$$

Theorem. (a) Under assumption (I), we have:

The restriction $L$ on $S$, the totality of rapidly decreasing functions, has a unique selfadjoint extension $H_{1}$, with $\mathfrak{D}\left(H_{1}\right)=\mathscr{D}_{L^{2}}^{2}\left(R^{n}\right)$. The absolutely continuous part of $H_{1}$ is unitarily equivalent to $H_{0}$.

(b) Assuming further (II), we have:

The singular spectrum of $H_{1}$ consists of non zero eigenvalues of finite multiplicity and possibly zero. Negative eigenvalues have not a finite limiting point. Zero is the only possible finite limiting point for positive eigenvalues if they exist.

Now we resume results of Kuroda. Let $H_{j}(j=0,1)$ be selfadjoint in a separable Hilbert space $\mathfrak{S}, R_{j}(z) \equiv\left(H_{j}-z\right)^{-1}$ be its resolvent for nonreal $z$. Let $\sigma\left(H_{j}\right)$ be its spectrum. Let $\mathfrak{S}_{j, a_{c}}\left(\mathfrak{S}_{j, s}\right)$ be the subspace of absolute continuity (of singularity) with respect to $H_{j}$. These concepts have been defined in Chapt. X of [1]. Then $\mathfrak{S}_{j, a c}$ and $\mathfrak{E}_{j, s}$ are closed linear subspaces of $\mathfrak{L}_{2}$, are orthogonal complements to each other and reduce $H_{j}$. If $\mathfrak{S}_{j, a c}=\mathfrak{S}_{\mathcal{E}}, H_{j}$ is said to be absolutely continuous. $H_{j, a c}\left(H_{j, s}\right)$ be the restriction of $H_{j}$ to $\mathfrak{S}_{j, a c}\left(\mathfrak{S}_{j, s}\right)$. The set $\sigma\left(H_{j, a_{c}}\right)\left(\sigma\left(H_{j, s}\right)\right)$ is the absolutely continuous (the singular) spectrum of $H_{j}$ and is denoted by $\sigma_{a_{c}}\left(H_{j}\right)\left(\sigma_{s}\left(H_{j}\right)\right)$. We 
denote by $\mathfrak{B}$ the space of all bounded linear operators from $\mathfrak{S}$ into $\mathfrak{S}$ having the uniform operator topology. For any linear operator $T$ its domain (or range) is represented as $\mathfrak{D}(T)$ (or $\mathfrak{R}(T)$ ).

Consider the following conditions on $H_{j}$.

(K. 1) $\mathfrak{D}\left(H_{1}\right)=\mathfrak{D}\left(H_{0}\right)=\mathfrak{D}$.

(K.2) There exist linear operators $A$ and $B$ such that: (a) $A$ lies in $\mathfrak{B}$, and is invertible with the range being dense in $\mathfrak{S},(b) \mathfrak{D}(B)$ $\supset \Re\left(R_{0}(z) A\right)$ for any nonreal $z,(c)\left(H_{1}-H_{0}\right) u=A B u$ for $u \in \mathfrak{D}(B) \cap \mathfrak{D}$.

(K. 3) $S(z) \equiv A^{*}\left\{R_{0}(z)-R_{0}(\bar{z})\right\} A$ lies in $\mathfrak{B}, \lim _{\varepsilon \downarrow 0} S(\lambda+i \varepsilon)=S(\lambda)$ exists in $\mathfrak{B}$, and this convengence is locally uniform in $\lambda$ of the real axis.

(K. 4) $Q(z) \equiv B R_{0}(z) A$ is in $\mathfrak{B}$, and completely continuous for nonreal $z$.

(K. 5) $\lim _{\varepsilon \neq 0} Q(\lambda \pm i \varepsilon)=Q(\lambda \pm i 0)$ exists in $\mathfrak{B}$ for any real $\lambda$, and $Q(z)$ is a $\mathfrak{B}$-valued continuous function on either the upper or the lower half-plane, including the corresponding edge of the real axis.

(K. 6) The operator $\left\{\frac{1}{2 \pi i} S(\lambda)\right\}^{1 / 2}$ is Hölder continuous with Hölder exponent $\theta>1 / 2$ on a closed interval $I$ of real axis, and $Q(z)$ is also Hölder continuous with exponent $\theta$ on $I^{\vdash}$ or $I^{-}$, where $I^{+}=\{z: \operatorname{Re} z \in I, \operatorname{Im} z \geqslant 0\}$ and $I^{-}=\{z: \operatorname{Re} z \in I, \operatorname{Im} z \leqslant 0\}$.

We can deduce the following theorem as a corollary of Kuroda's theory ([2], [3]). The outline of the proof will be sketched in $\S 3$.

Theorem K. Under conditions (K.1) to (K.5) we have:

$\mathbf{1}^{\circ} \quad H_{0}$ is absolutely continuous.

$2^{\circ} \quad \sigma_{a c}\left(H_{1}\right)=\sigma\left(H_{0}\right)$, and $\sigma_{s}\left(H_{1}\right)$ is a closed null set.

$3^{\circ}$ There exist wave operators $W_{ \pm} \in \mathfrak{B}$ such that: $W_{ \pm}^{*} W_{ \pm}=1$, $W_{ \pm} W_{ \pm}^{*}=P_{1}, H_{1} W_{ \pm}=W_{ \pm} H_{0}$ and $W_{ \pm}=\underset{t \rightarrow P_{\infty}}{\mathrm{s}-\lim } e^{i t H_{1}} e^{-i t H_{0}}$ where 1 is the identity operator, and where $P_{1}$ is the projection to $\mathfrak{S}_{1, a c}$.

Assuming further (K.6), we obtain:

$4^{\circ} \sigma_{s}\left(H_{1}\right) \cap I$ consists of at most a countable number of eigenvalues of finite multiplicity which have no accumulation point interior to $I$. 


\section{$\S 2$. Proof of the Theorem}

First we write

$$
L=-\Delta+\alpha(x)\left(\sum_{j=1}^{n} \beta_{j}(x) \frac{\partial}{\partial x_{j}}+\beta_{0}(x)\right)
$$

where $\alpha(x) \beta_{0}(x)=q^{\prime}(x)=\sum_{j=1}^{n}\left(\frac{\partial}{i \partial x_{j}} b_{j}(x)+b_{j}(x)^{2}\right)+q(x)$ and $\alpha(x) \beta_{j}(x)=$ $-2 i b_{j}(x)(1 \leqslant j \leqslant n)$. Define $(A f)(x) \equiv \alpha(x) \cdot f(x),\left(B_{0} f\right)(x) \equiv \beta_{0}(x) f(x)$ $\left(B_{j} f\right)(x) \equiv \beta_{j}(x) \frac{\partial}{\partial x_{j}} f(x)(1 \leqslant j \leqslant n)$ then we have formally $H_{1}=H_{0}+A B$ for $B=\sum_{j=1}^{n} B_{j}$.

From now on we will take $\alpha(x)=(1+|x|)^{-a}$, then the operator $A$ belongs to $\mathfrak{B}$ and satisfies conditions (a) of (K. 2). Operators $B_{j}$ with domains $\left\{f(x) \mid f(x) \in L^{2},\left(B_{j} f\right)(x) \in L^{2}\right\}$ have closed extensions, which are also denoted by $B_{j}$. For the proof of the first part of (a), we have only to show that $\mathfrak{D}\left(H_{0}\right)$ is contained in $\mathfrak{D}(A B)$ and that for any $\varepsilon>0$ there exists $C_{\varepsilon}>0$ such that for any $u \in \mathfrak{D}\left(H_{0}\right)$

$$
\|A B u\| \leqslant \varepsilon\left\|H_{0} u\right\|+c_{\varepsilon}\|u\|
$$

(Chapt. V of [1]).

Assume that $D\left(B_{j}\right) \supset D\left(H_{0}{ }^{\mu}{ }_{j}\right)$ for $\mu_{j}$ with $0<\mu_{j}<1$, then we have for $u \in D\left(H_{0}\right)$ and $k>1$

$$
\begin{aligned}
\left\|B_{j} u\right\| & =\left\|B_{j} R_{0}(-1)^{\mu_{j}}\left(H_{0}+1\right)^{\mu_{j}} R_{0}(-k)\left(H_{0}+k\right) u\right\| \\
& \leqslant\left\|B_{j} R_{0}(-1)^{\mu_{j}}\right\| \cdot i \mid\left(H_{0}+1\right)^{\mu_{j}} R_{0}(-k) \| \cdot\left\{\left\|H_{0} u\right\|+k\left\|_{i}^{\prime} u\right\|\right\} .
\end{aligned}
$$

By the closed graph theorem, $B_{j} R_{0}(-1)^{\mu_{j}}$ is bounded. Noticing that for $k>1$

$$
\left\|\left(H_{0}+1\right)^{\mu} R_{0}(-k)\right\| \leqslant k^{\mu_{j}-1},
$$

we have the estimate that there exists $C_{\varepsilon}>0$

$$
\left\|B_{j} u\right\| \leqslant \varepsilon\left\|H_{0} u\right\|+C_{\varepsilon} i \mid u \|
$$

for $\varepsilon>0$ and $u \in \mathfrak{D}\left(H_{0}\right)$. Since $A$ is bounded, we will obtain the desired statements if $\mathfrak{D}\left(B_{j}\right) \supset \mathfrak{D}\left(H_{0}{ }^{\mu}{ }_{j}\right)$.

Now we will show the validity of this inclusion for $1 \leqslant j \leqslant n$. Since $p_{1}>n$ (condition I), we can choose $\mu_{j}$ such that $p_{1}>\frac{n}{2 \mu_{j}-1}$ and 
$<\mu_{j}<1$. If $u \in D\left(H_{0}^{\mu_{j}}\right)$, the Fourier transform of $u(x), \hat{u}(\xi)$ satisfies

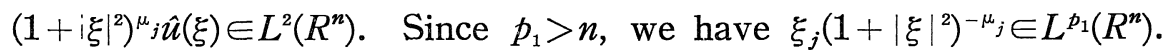
As $\xi_{j} \hat{u}=\xi_{j}\left(1+|\xi|^{2}\right)^{-\mu_{j}}\left(1+|\xi|^{2}\right)^{\mu_{j}} \hat{u}, \xi_{j} \hat{u} \in L_{q}\left(R^{n}\right)$ for $\frac{1}{q}=\frac{1}{p_{1}}+\frac{1}{2}$. Therefore $\frac{\partial}{\partial x_{j}} u \in L^{q^{\prime}}$ with $\frac{1}{q^{\prime}}=1-\frac{1}{q}=\frac{1}{2}-\frac{1}{p_{1}}$. As $\beta_{j} \in L^{p_{1}}$ and $\frac{1}{p_{1}}+\frac{1}{q^{\prime}}=\frac{1}{2}$, we have $B_{j} u=\beta_{j} \frac{\partial}{\partial x_{j}} u \in L^{2}\left(R^{n}\right)$.

Next we treat the case $j=0$. Since $p_{2}>\frac{n}{2}$, we can choose $\mu_{0}$ satisfying $p_{2}>\frac{n}{2 \mu_{0}}$ and $0<\mu_{0}<1$. Then $\left(1+|\xi|^{2}\right)^{-\mu_{0}} \in L^{p_{2}}\left(R^{n}\right)$. As $\hat{u}=\left(1+|\xi|^{2}\right)^{-\mu_{0}}\left(1+|\xi|^{2}\right)^{\mu_{0}} \hat{u}, \hat{u} \in L^{q}\left(R^{n}\right)$ for $\frac{1}{q}=\frac{1}{p_{2}}+\frac{1}{2}$. Therefore $u \in L^{q^{\prime}}\left(R^{n}\right)$ with $\frac{1}{q^{\prime}}=1-\frac{1}{q}=\frac{1}{2}-\frac{1}{p_{2}}$. And finally we have $B_{0} u=\beta_{0} u$ $\in L^{2}\left(R^{n}\right)$. Thus the first part of (a) has been proved.

Now we start to check conditions (K.1) to (K.5). For $r>0$ we define

with

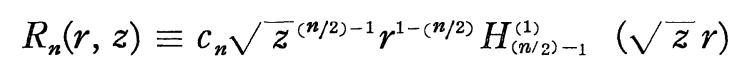

$$
c_{n}=i 2^{-(n / 2)-1} \pi^{1-(n / 2)}, \quad \operatorname{Im} \sqrt{z} \geqslant 0
$$

and the $\nu$-th Hankel function of first kind $H_{\nu}{ }^{(1)}(\zeta)$. Then it holds that for a nonreal or negative number $z$,

$$
\left(H_{0}-z\right)^{-1} f(x)=\int_{R^{n}} R_{n}(|x-y|, z) f(y) d y .
$$

The following asymptotic representations are well known:

(H. 1) $H_{\nu}{ }^{(1)}(\zeta)=-\pi^{-1} 2^{-\nu} \Gamma(\nu) \zeta^{-\nu}+0\left(\zeta^{-\nu}\right)$ as $|\zeta| \rightarrow 0$ with $\operatorname{Im} \zeta \geqslant 0$,

(H. 2) $\quad H_{\nu}^{(1)}(\zeta)=\sqrt{2}(\pi \zeta)^{-(1 / 2)} e^{i(\zeta-(2 v+1)(\pi / 4))}+0\left(\zeta^{-(3 / 2)}\right)$

$$
\text { as }|\zeta| \rightarrow \infty \text { with } \operatorname{Im} \zeta \geqslant 0 \text {. }
$$

Taking $\phi(\zeta) \in C^{\infty}$ such that $\phi(\zeta)=1$ for $|\zeta| \leqslant 1$ and $\phi(\zeta)=0$ for $|\zeta| \geqslant 2$, we have

$$
\begin{aligned}
R_{n}(r, z) & =\phi(\sqrt{z} r) R_{n}(r, z)+(1-\phi(\sqrt{z} r)) R_{n}(r, z) \\
& =\frac{S_{n}(r, z)}{r^{n-2}}+\frac{S_{n}(r, z)}{r^{(n-1) / 2}} .
\end{aligned}
$$

Let $\Pi$ be the complex plane which has a cut along the positive real axis from 0 to $\infty$, including both edges of the cut. Then $S_{n}{ }^{(k)}(r, z)$ 
can be regarded to be continuous in $(r, z) \in(0, \infty) \times(\Pi-\{0\})$. From (H. 1) and (H. 2) it holds that:

$$
\left|S_{n}{ }^{(1)}(r, z)\right| \leqslant \text { const, } S_{n}{ }^{(1)}(r, z)=0 \text { for }|\sqrt{z} r| \geqslant 2,
$$

and that :

$$
\left|S_{n}{ }^{(2)}(r, z)\right| \leqslant \mathrm{const}|z|^{(n-2) / 4}, S_{n}^{(2)}(r, z)=0 \text { for }|\sqrt{z} r| \leqslant 1 .
$$

By the identity $\frac{d}{d \bar{\zeta}}\left(\zeta^{-\nu} H_{\nu}{ }^{(1)}(\zeta)\right)=-\zeta^{-\nu} H_{\nu+1}^{(1)}(\zeta)$, we deduce that for $r=\left(\sum_{j=1}^{n} x_{j}^{2}\right)^{1 / 2}$

$$
\begin{aligned}
\frac{\partial}{\partial x_{j}} R_{n}(r, z) & =-\frac{c_{n}}{c_{n+2}} \cdot \frac{x_{j}}{r} \cdot R_{n+2}(r, z) \\
& =\frac{S_{n, j}{ }^{(1)}(x, z)}{r^{n-1}}+\frac{S_{n, j^{(2)}}(r, z)}{r^{(n-1) / 2}}
\end{aligned}
$$

where $S_{n, j}{ }^{(k)}(x ; z) \equiv-c_{n} / c_{n ! 2} \cdot \frac{x_{j}}{r} \cdot S_{n ! 2}{ }^{(k)}(r, z) \quad(k=1,2,1 \leqslant j \leqslant n)$.

These functions satisfy the estimates of the same type as $S_{n / 2}{ }^{(k)}$. Define $\Pi_{N}^{+} \equiv\left\{z: N^{-1} \leqslant|z| \leqslant N, \operatorname{Im} z \geqslant 0\right\}$ for $N>1$, and similarly $\Pi_{N}^{-}$ for $\operatorname{Im} z \leqslant 0$. Noticing that $S_{n}{ }^{(1)}(r, z)=r^{n-2} \phi(\sqrt{z} r) R_{n}(r, z)$ and that $\left.S_{n}{ }^{(2)}(r, z)=r^{(n-1) / 2}(1-\phi \sqrt{z} r)\right) R_{n}(r, z)$, using the identity $\frac{d}{d \zeta}\left(\zeta^{\nu} H_{\nu}{ }^{(1)}(\zeta)\right)$ $=\zeta^{\nu} H_{\nu-1}{ }^{(1)}(\zeta)$, we have the following estimates :

$$
\left|\frac{\partial}{\partial z} S_{n}{ }^{(1)}(r, z)\right| \leqslant c_{N}, \text { and }\left|\frac{\partial}{\partial z} S_{n}^{(2)}(r, z)\right| \leqslant c_{N} r
$$

for $z \in \Pi_{N}^{ \pm}$where $c_{N}$ is some constant depending only on $N$. Therefore we have

$$
\left|\frac{\partial}{\partial z} S_{n, j}{ }^{(1)}(x, z)\right| \leqslant{c^{\prime}}_{N} \quad \text { and } \quad\left|\frac{\partial}{\partial z} S_{n, j}{ }^{(2)}(x, z)\right| \leqslant{c^{\prime}}_{N} r
$$

for $z \in \Pi_{N}^{ \pm}$and $1 \leqslant j \leqslant n$.

Letting $S_{n, 0}{ }^{(k)}(x, z) \equiv S_{n}{ }^{(k)}(|x|, z)$, we define integral kernels :

$$
Q_{j}{ }^{(k)}(x, y ; z)=\frac{\beta_{j}(x) S_{n, j}{ }^{(k)}(x-y, z) \alpha(y)}{|x-y|^{\lambda_{j, k}}} \quad(k=1,2,0 \leqslant j \leqslant n)
$$

where $\quad \lambda_{0,1}=n-2, \lambda_{j, 1}=n-1(1 \leqslant j \leqslant n)$ and $\lambda_{j, 2}=\frac{n-1}{2} \quad(0 \leqslant j \leqslant n)$ and integral operators : 


$$
\left(Q_{j}{ }^{(k)}(z) f\right)(x)=\int_{R^{n}} Q_{j}^{(k)}(x, y ; z) f(y) d y .
$$

Formally we have $Q(z)=B R_{0}(z) A=\sum_{j=0}^{n} \sum_{k=1}^{2} Q_{j}{ }^{(k)}(z)$. We need :

Lemma. For a given $\lambda$ satisfying $n>\lambda>0$, assume that there exist $p, q$ such that $\frac{1}{p},-\frac{1}{q}<\frac{1}{2}$ and $\frac{1}{p}+\frac{1}{q}=1-\frac{\lambda}{n}$. Consider an integral operator: $\left(K_{\lambda} f\right)(x)=\int_{R^{n}} \frac{\beta(x) \gamma(x, y) \alpha(y)}{|x-y|^{\lambda}} f(y) d y$, where $\gamma(x, y)$ is continuous and bounded on $R^{2 n}-\{x=y\}$.

If $\alpha(x) \in L^{p}\left(R^{n}\right)$ and $\beta(x) \in L^{q}\left(R^{n}\right)$, then $K_{\lambda}$ is a bounded operator in $L^{2}\left(R^{n}\right)$ satisfying

$$
\left\|K_{\lambda}\right\|_{\mathfrak{B}} \leqslant C(p, q)\|\gamma\|_{\infty} \cdot\|\beta\|_{q} \cdot \dot{i} \mid \alpha \|_{p}
$$

where $\|\gamma\|_{\infty}=\sup _{x_{\mp} y}|\gamma(x, y)|,\|u\|_{p}=\left(\int_{R_{n}}|u(x)|^{p} d x\right)^{1 / p}$.

Moreover if we assume further that $\alpha(x) \in L^{p^{\prime}}\left(R^{n}\right)$ and $\beta(x) \in$ $L^{q^{\prime}}\left(R^{n}\right)$ where $p^{\prime}$ and $q^{\prime}$ satisfy $\frac{1}{2}-\frac{\lambda}{n} \leqslant \frac{1}{p^{\prime}}<\frac{1}{2}$ and $\frac{1}{p^{\prime}}+\frac{1}{q^{\prime}}<1-\frac{\lambda}{n}$, then $K_{\lambda}$ is completely continuous (due to Kuroda, see lemma 5.3 of [4]).

Proof. Sobolev's inequality shows that $\left|\int_{R^{n}} \int_{R^{n}} \frac{g(x) f(y)}{|x-y|^{\lambda}} d x d y\right|$ $\leqslant C(P, Q)\|g\|_{Q}\|f\|_{P}$ for $P>1, Q>1, \frac{1}{P}+\frac{1}{Q}>1$ and $\lambda=n\left(2-\frac{1}{P}-\frac{1}{Q}\right)$ ([6]). Let $\frac{1}{P}=\frac{1}{2}+\frac{1}{p}$ and $\frac{1}{Q}=\frac{1}{2}+\frac{1}{q}$. Substituting the above inequility for $g=\beta v$ and $f=\alpha u$ where $u, v \in L^{2}\left(R^{n}\right)$, we have readily the first part of the lemma.

Let $\chi_{N}(x)$ be the characteristic function of $D_{N}=\{x|| x \mid \leqslant N\}$. Define $\left(K_{\lambda}{ }^{(N, M)} f\right)(x) \equiv \int_{R^{n}} \frac{\chi_{M}(x) \beta(x) \gamma(x, y) \alpha(y) \chi_{N}(y)}{|x-y|^{\lambda}} f(y) d y$.

By our assumption on $p^{\prime}$ and $q^{\prime}$, Kondrašev's Theorem ([7]) asserts that an integral kernel, $\frac{\gamma(x, y)}{|x-y|^{\lambda}}$, determins a completely continuous integral operator from $L^{P^{\prime}}\left(D_{N}\right)$ to $L^{Q^{\prime}}\left(D_{N}\right)$ for $N<\infty$ with $\frac{1}{P^{\prime}}=\frac{1}{p^{\prime}}$ $+\frac{1}{2}$ and $\frac{1}{Q^{\prime}}=\frac{1}{2}-\frac{1}{q^{\prime}}$. Since we can regard $\alpha \cdot \chi_{N}$ as a bounded operator from $L^{2}\left(R^{n}\right)$ to $L^{P^{\prime}}\left(D_{N}\right)$ and $\beta \cdot \chi_{N}$ as that from $L^{Q^{\prime}}\left(D_{N}\right)$ to 
$L^{2}\left(R^{n}\right)$, we have that $K_{\lambda}^{(N, N)}$ is completely continuous from $L^{2}\left(R^{n}\right)$ to $\left(L^{2}\left(R^{n}\right)\right.$. But from the first part of the lemma, we have

$$
\begin{aligned}
\left\|K_{\lambda}-K_{\lambda}(N, N)\right\| & \leqslant\left\|K_{\lambda}-K_{\lambda}^{(\infty, N)}\right\|+\left\|K_{\lambda}^{(\infty, N)}-K_{\lambda}{ }^{(N, N)}\right\| \\
& \leqslant C(p, q)\left\{\left\|\left(1-\chi_{N}\right) \alpha\right\|_{p}\|\beta\|_{q}+\left\|\chi_{N} \alpha\right\|_{p}\left\|\left(1-\chi_{N}\right) \beta\right\|_{q}\right\} \\
& \rightarrow 0 \quad(\text { as } N \text { tends to } \infty) .
\end{aligned}
$$

Therefore $K_{\lambda}$ is completely continuous. Thus the lemma has been proved.

In the above lemma, if we take $\alpha(x)=(1+|x|)^{-a}$ where $a>\frac{n}{2}$, and $\beta(x) \in L^{q}$ where $q$ satisfies $\frac{1}{2}-\frac{\lambda}{n}<\frac{1}{q}<\frac{1}{2}$ for $\frac{1}{2}-\frac{\lambda}{n}>0$ and $\frac{1}{q}<1-\frac{\lambda}{n}$ for $\frac{1}{2}-\frac{\lambda}{n} \leqslant 0$, then $\alpha(x) \in L^{p}$ for any $p$ with $\frac{1}{p} \leqslant \frac{1}{2}$. Since we can choose $p$ and $p^{\prime}$ appropriately, the above lemma asserts that $K_{\lambda}$ is completely continuous in $L^{2}\left(R^{n}\right)$. More precisely, if $\beta(x)$ $\in L^{q_{j}}$ where $q_{j}$ satisfy that:

$$
\begin{aligned}
& \text { for } \lambda_{1}=n-1, \quad q_{1}>n \text {, } \\
& \text { for } \lambda_{2}=n-2, \text { if } n=3 \text {, then } 6>q_{2}>2 \text {, } \\
& \text { if } n>3 \text {, then } q_{2}>\frac{n}{2} \text {, } \\
& \text { for } \lambda_{3}=\frac{n-1}{2}, 2 n>q_{3}>2 \text {, }
\end{aligned}
$$

then $K_{\lambda_{j}}$ are completely continuous. Therefore $Q_{j}{ }^{(k)}(z)$ are completely continuous if $\beta_{0}(x) \in L^{q_{2}} \cap L^{q_{3}}$ and if $\beta_{j}(x) \in L^{q_{1}} \cap L^{q_{2}}(1 \leqslant j \leqslant n)$. These being assumed in condition (I), we will obtain (K.4) if the operator $Q(z)$, treated above, coincides with $B R_{0}(z) A$. We will show this fact. From Sobolev's inequality it holds that if $f \in L^{2}$, then

$$
\int_{R^{n}} \frac{\partial}{\partial x_{j}} R_{n}(|x-y|, z) \alpha(y) f(y) d y \in L^{p} \quad \text { for } \quad 1 \geqslant \frac{1}{p} \geqslant \frac{1}{2} .
$$

This implies that $\mathscr{D}(B) \supset \Re\left(R_{0}(z) A\right) \quad((\mathrm{b})$ of (K.2)) and that our integral operator $Q(z)$ is equal to $B R_{0}(z) A$.

Naturally we can define $Q(\lambda \pm i 0)$ for $\lambda>0$ as the integral operator with the kernel $\sum_{j, k} Q_{j}{ }^{(k)}(x, y ; \lambda \pm i 0)=\lim _{\varepsilon \downarrow 0} \sum_{j, k} Q_{j}^{(k)}(x, y ; \lambda \pm i \varepsilon)$ for $x \neq y$. Also $Q(0)$ can be defined by putting $R_{n}(r, 0)=(n-2)^{-1}(2 \pi)^{-(n / 2)}$ 
$\times \Gamma\left(\frac{n}{2}\right) r^{2-n}$. We denote by $Q^{(N)}(z)$ the operator which is obtained by the replacing $\beta_{j}(x)$ by $\chi_{N}(x) \beta_{j}(x)$ and $\alpha(x)$ by $\chi_{N}(x) \alpha(x)$ in $Q(z)$. As in the proof of the lemma, we can show $\lim _{N \rightarrow \infty} Q^{(N)}(z)=Q(z)$ in $\mathfrak{B}$ uniformly in $z$ belonging to a bounded set of either the upper or the lower half-plane including the real axis. The asymptotic formula (H. 1) asserts that if $\operatorname{Im} z_{1}, \operatorname{Im} z_{2} \geqslant 0$ (or $\leqslant 0$ ) and if $\left|z_{1}\right|,\left|z_{2}\right|$ and $|r| \leqslant N$, we have $R_{n}\left(r, z_{1}\right)-R_{n}\left(r, z_{2}\right)=\frac{o(1)}{r^{n-2}}$ uniformly as $\left|z_{1}-z_{2}\right|$ tends to zero. This assures that $Q^{(N)}(z)$ is a $\mathfrak{B}$-valued continuous function on $\operatorname{Im} z \geqslant 0$ (or $\operatorname{Im} z \leqslant 0$ ). Therefore $Q(z)$ is a $\mathfrak{B}$-valued continuous function, which implies (K. 5).

As for (K. 3), we must consider integral operators $A^{(k)}(z)$ with kernels : $A^{(k)}(x, y ; z)=\frac{\overline{\alpha(x)} S_{n}{ }^{(k)}(x-y, z) \alpha(y)}{|x-y|^{\lambda_{0, k}}}(k=1,2)$. Since $A^{(k)}(z)$ behave similarly to $Q_{0}{ }^{(k)}(z)$, we omit to describe the check of this condition.

Finally we will show that (K.6) holds under conditions (I) and (II). Let $I$ be a closed interval on the real axis which does not contain zero. Since $\frac{\partial}{\partial z} S_{n, j}{ }^{(1)}(x, z)$ is bounded in $z \in \Pi_{N}^{ \pm}, Q_{j}^{(1)}(z)$ $(0 \leqslant j \leqslant n)$ is Lipschitz continuous on $I^{ \pm}$. Next we notice that for $z_{1}, z_{2} \in \Pi_{N}^{+}$

$$
\begin{aligned}
\frac{\left|S_{n, j^{(2)}}\left(x, z_{1}\right)-S_{n, j^{(2)}}\left(x, z_{2}\right)\right|^{\theta}}{|r|^{\theta}} & =\frac{1}{r^{\theta}}\left|\int_{z_{2}}^{z_{2}} \frac{\partial}{\partial z} S_{n, j}{ }^{(2)}(x, z) d z\right|^{\theta} \\
& \leqslant \text { const }\left|z_{1}-z_{2}\right|^{\theta}
\end{aligned}
$$

since $\left|\frac{\partial}{\partial z} S_{n, j}^{(2)}\right| \leqslant C_{N}|r|$. Putting $\theta=\frac{1}{2}+\varepsilon$ and $\lambda_{4}=\frac{n}{2}-1-\varepsilon(\varepsilon>0)$, the integral kernel of $Q_{j}{ }^{(2)}\left(z_{1}\right)-Q_{j}{ }^{(2)}\left(z_{2}\right)$ is estimated by const $\left|z_{1}-z_{2}\right|^{(1 / 2)+\varepsilon} \frac{\beta_{j}(x) \alpha(y)}{|x-y|^{\lambda_{4}}}$ for $z_{1}, z_{2} \in \Pi_{N}^{ \pm}$. By the lemma if $\beta_{j} \in L^{q_{4}}$ with $\frac{1+\varepsilon}{n}<\frac{1}{q_{4}}<\frac{1}{2}$, the above kernel defines a bounded operator $\in \mathfrak{B}$. Since we can choose $\varepsilon$ arbitrarily small, condition (II) asserts that $Q_{j}{ }^{(2)}(z)$ is Hölder continuous on $\Pi_{N}^{ \pm}$with the exponent greater than $1 / 2$. The Hölder continuity of $\left\{\frac{1}{2 \pi i} S(\lambda)\right\}^{1 / 2}$ has been shown by Kuroda in case $n=3([2])$. He used the spherical coordinate representation 
of $S(\lambda)$. His method is also valid for $n>3$ if we take the $n$-dimensional spherical harmonics instead of the 3-dimensional.

Since $\lim _{\substack{\lambda<0 \\ \lambda \rightarrow 0}} Q(\lambda)=Q(0)$ holds, applying Theorem 1 of [5] to our case, we know that negative eigenvalues do not accumulate at zero. Another conclusions of our theorem follow from the result of Kuroda.

\section{§3. Remarks}

\section{Outline of the proof of Theorem $K$.}

By (K.1) we have the second resolvent equation:

$$
R_{1}(z)=R_{0}(z)-R_{1}(z)\left(H_{1}-H_{0}\right) R_{0}(z) .
$$

Since $A$ is bounded ((a) of (K.2)), it holds that

$$
R_{1}(z) A=R_{0}(z) A-R_{1}(z)\left(H_{1}-H_{0}\right) R_{0}(z) A .
$$

Noticing that $\mathfrak{D}(B) \cap \mathfrak{D} \supset \mathfrak{R}\left(R_{0}(z) A\right)$ ((b) of (K. 2)), we have

$$
\begin{aligned}
\left(H_{1}-H_{0}\right) R_{0}(z) A & =A B R_{0}(z) A & & ((\mathrm{c}) \text { of }(\mathrm{K} .2)) \\
& =A Q(z) & & ((\mathrm{K} .3)) .
\end{aligned}
$$

Therefore the following identity is obtained.

$$
R_{1}(z) A(1+Q(z))=R_{0}(z) A .
$$

Define $G_{0}(z) \equiv 1+Q(z)$ for $\operatorname{Im} z \neq 0$. If $u \in \mathfrak{S}$ satisfies that $u+Q(z) u=0$ then $v=R_{0}(z) A u$ satisfies $H_{1} v=z v$. Since $\operatorname{Im} z \neq 0$, self-adjointness of $H_{1}$ implies that $v=0$. Hence $u=0$. Moreover $Q(z)$ is completely continuous $((\mathrm{K} .4))$. Therefore there exists a bounded inverse of $(1+Q(z))$. We write $G_{1}(z) \equiv(1+Q(z))^{-1}$ for $\operatorname{Im} z \neq 0$. Then we have from (3.1) identities

$$
\left\{\begin{array}{l}
R_{0}(z) A G_{1}(z)=R_{1}(z) A, \\
R_{1}(z) A G_{0}(z)=R_{0}(z) A .
\end{array}\right.
$$

Let $S_{j}(z) \equiv A^{*}\left\{R_{j}(z)-R_{j}(\bar{z})\right\} A$ for $j=0,1$ and $\operatorname{Im} z \neq 0$. We have

$$
\begin{aligned}
& A^{*}\left\{R_{j}(z)-R_{j}(\bar{z})\right\} A=(z-\bar{z}) A^{*} R_{j}(\bar{z}) R_{j}(z) A \\
& =(z-\bar{z})\left(R_{j}(z) A\right)^{*} R_{j}(z) A
\end{aligned}
$$




$$
\begin{aligned}
& =(z-\bar{z})\left(R_{k}(z) A G_{j}(z)\right) * R_{k}(z) A G_{j}(z) \quad(\text { by }(3.2)) \\
& =G_{j}(z) * A^{*}\left\{R_{k}(z)-R_{k}(\bar{z})\right\} A G_{j}(z) .
\end{aligned}
$$

This relation is rewritten as

$$
S_{j}(z)=G_{j}(z) * S_{k}(z) G_{j}(z)
$$

where $(j, k)=(0,1)$ or $(1,0)$.

On the other hand it is well known that for $\alpha$ and $\beta$ with $-\infty \leqslant \alpha<\beta \leqslant \infty$,

$$
\int_{\infty}^{\beta} d E_{j}(\lambda)=\underset{\varepsilon \downarrow 0}{\mathrm{~s}-\lim _{\varepsilon}} \frac{1}{2 \pi i} \int_{\infty}^{\beta}\left(R_{j}(\lambda+i \varepsilon)-R_{j}(\lambda-i \varepsilon)\right) d \lambda .
$$

From this identity we obtain that for $u, v \in \mathfrak{S}$,

$$
\int_{\alpha}^{\beta} d\left(E_{j}(\lambda) \Lambda u, A v\right) d \lambda=\frac{1}{2 \pi i} \lim _{\varepsilon \downarrow 0} \int_{\alpha}^{\beta}\left(S_{j}(\lambda+i \varepsilon) u, v\right) d \lambda .
$$

For $j=0$ condition (K. 3) assures that

$$
\int_{\alpha}^{\beta} d\left(E_{0}(\lambda) A u, A v\right)=\frac{1}{2 \pi i} \int_{\alpha}^{\beta}(S(\lambda) u, v) d \lambda .
$$

By the continuity of $S(\lambda)$, we can conclude that for any measurable set $\omega$ of the real line

$$
A^{*} E_{0}(\omega) A=\frac{1}{2 \pi i} \int_{\omega} S(\lambda) d \lambda
$$

(the integration can be performed in the operator topology). This identity implies that $\mathfrak{R}(A)$ is contained in $\mathfrak{S}_{0, a c}$. But $\Re(A)$ is dense in $\mathfrak{S}$ and $\mathfrak{S}_{0, a c}$ is closed. We know $\mathfrak{S}_{0, a_{c}}=\mathfrak{S}_{\mathcal{C}}$. This means conclusion $1^{\circ}$ of Theorem $\mathrm{K}$.

By (K. 5) there exists

$$
G_{0}(\lambda \pm i 0)=\lim _{\varepsilon \downarrow 0} G_{0}(\lambda \pm i \varepsilon)=1+Q(\lambda \pm i 0) .
$$

Since $G_{0}(z)-1=Q(z)$ is completely continuous for $\operatorname{Im} z \neq 0((\mathrm{~K} .4))$, $G_{0}(\lambda \pm i 0)-1$ is also completely continuous. And $G_{1}(z)=G_{0}(z)^{-1}$ for $\operatorname{Im} z \neq 0$. Therefore lemma 6.2 of [3] (or lemma 5.2 of [2]) assures that there exists a bounded inverse of $G_{0}(\lambda \pm i 0), G_{1}(\lambda \pm i 0)$, for almost every $\lambda$ of the real axis. We denote by $e$ the subset of the real line which consists of points $\lambda$ such that $G_{0}(\lambda \pm i 0)$ can not be 
invertible. Since $G_{0}(\lambda \pm i 0)$ is continuous in $\lambda$ of the real axis, the complement of $e, e^{\prime}$ is open. Moreover $G_{1}(z)$ is continuous on $e^{+}$and $e^{\prime-}$ respectively, where $e^{\prime+}\left(e^{\prime-}\right)=\left\{z: \operatorname{Re} z \in e^{\prime}, \operatorname{Im} z \geqslant 0(\operatorname{Im} z \leqslant 0)\right\}$. Therefore we have for $\lambda \notin e$

$$
G_{1}(\lambda \pm i 0)=\lim _{\varepsilon \downarrow 0} G_{1}(\lambda \pm i \varepsilon) \quad \text { in } \mathfrak{B} .
$$

Define $S_{1}(\lambda \pm i 0) \equiv \pm G_{1}(\lambda \pm i 0) * S(\lambda) G_{1}(\lambda \pm i 0)$ for $\lambda \notin e$. By (3.3) it is obtained that for $\lambda \notin e$

$$
S_{1}(\lambda \pm i 0)=\lim _{\varepsilon \downarrow 0} S_{1}(\lambda \pm i \varepsilon) \quad \text { in } \mathfrak{B} .
$$

If $(\alpha, \beta) \cap e=\phi$, the equality (3.4) implies that for $u, v \in \mathfrak{S}$

$$
\int_{\alpha}^{\beta} d\left(E_{1}(\lambda) A u, A v\right)=\frac{ \pm 1}{2 \pi i} \int_{\alpha}^{\beta}\left(S_{1}(\lambda \pm i 0) u, v\right) d \lambda .
$$

From this identity we conclude that for any closed set $\omega$ such that $\omega \cap e=\phi$, it holds that

$$
A^{*} E_{1}(\omega) A=\underset{2 \pi i}{ \pm 1} \int_{\omega} S_{1}(\lambda \pm i 0) d \lambda .
$$

Using this relation we can conclude that $\sigma_{a_{c}}\left(H_{1}\right)=\sigma\left(H_{0}\right)$, and that $\sigma_{s}\left(H_{1}\right) \subset e$. This means conclusion 2 of Theorem $\mathrm{K}$. We can also deduce conclusion $3^{\circ}$ of Theorem $\mathrm{K}$ as in the proof of Theorems 5.1 to 5.4 of [3] (or Theorem 4.1 of [2]). Finally conclusion $4^{\circ}$ of Theorem $\mathrm{K}$ follows from Theorem 7.1 of [3] (Professor Kuroda kindly informed the author that the condition of Hölder continuity of $Q(z)$ was missing in [3]).

\section{Eigenfunction expansions.}

Under conditions (K.1) to (K.5), we have more concrete knowledge concerning the spectral representation of $H_{1, a c}$ by Kuroda's criterion. Consider the following integral equation:

$$
\begin{aligned}
\varphi^{ \pm}(x, \xi) & =e^{i x \xi}-\int_{R^{n}} \phi^{ \pm}(y, \xi) \\
\times & \left\{2 i \sum_{j=1}^{n}\left(b_{j}(y) \frac{\partial}{\partial y_{j}}+\frac{\partial b_{j}(y)}{\partial y_{j}}+b_{j}(y)^{2}\right)+q(y)\right\} \\
\times & R_{n}\left(|x-y|:|\xi|^{2} \mp i 0\right) d y
\end{aligned}
$$


If $|\xi|^{2} \notin \sigma_{s}\left(H_{1}\right)$, we can find the unique $(x, \xi)$-measurable solution of $\varphi_{1}^{ \pm}(x, \xi)$ with $\int_{R^{n}}(1+|x|)^{-2 a}\left|{\varphi_{1}}^{ \pm}(x, \xi)\right|^{2} d x<\infty$. Define $\left(F^{ \pm} f\right)(\xi)=$ $(2 \pi)^{-(n / 2)} \int_{R^{n}} f(x) \varphi^{ \pm}(x, \xi) d x$ for $f$ with $(1+|x|)^{a} f \in L^{2}\left(R^{n}\right)$. Kuroda's abstract theorem (shown in [2]) asserts that $F$ can be extended to an isometric operator from $\mathfrak{S}_{1, a c}$ onto $L^{2}\left(R^{n}\right)$, satisfying $\left(F^{ \pm} P_{1} E_{1}(\lambda) f\right)(\xi)$ $=\chi_{\lambda}(\xi)\left(F^{ \pm} P_{1} f\right)(\xi)$ where $\chi_{\lambda}(\xi)$ is the characteristic function of $\left\{\left.\xi|| \xi\right|^{2} \leqslant \lambda\right\}$. Moreover if it holds that $(1+|x|)^{a} H_{1} f \in L^{2}$ for $f \in \mathcal{S}$, then we have for $|\xi|^{2} \notin \sigma_{s}\left(H_{1}\right),\left[\sum_{j=1}^{n}\left(\frac{\partial}{i \partial x_{j}}+b_{j}(x)\right)^{2}+q(x)\right] \varphi^{ \pm}(x, \xi)=$ $|\xi|^{2} \varphi^{ \pm}(x, \xi)$ in the sense of distribution. Some additional information of regularity of $\varphi^{ \pm}(x, \xi)$ will be obtained under appropriate conditions on $b_{j}$ and $q$.

3. After this work was completed, the author heard the work of Ikebe-Tayoshi ([8]) from Professor Ikebe. They treated a 3-dimensional Schrödinger operator : $L=-\Delta+\sum_{j, k=1}^{3} \alpha_{j, k}(x) \frac{\partial^{2}}{\partial x_{j} \partial x_{k}}+\sum_{k=1}^{3} \beta_{k} \frac{\partial}{\partial x_{k}}+\gamma$. They have established the unitary equivalence of $L$ and $-\Delta$ chiefly using the condition of $\alpha_{j k}, \beta_{k}$ and $\gamma \in L^{1}\left(R^{3}\right)$. Their method is based on the fact that if $R_{1}^{2}(z)-R_{0}^{2}(z)$ is an operator of the trace class, then the part (a) of our Theorem follows from Kato's criterion (Chapt. X of [1]).

\section{References}

[1] Kato, T., Perturbation theory for linear operators, Springer, Berlin, 1966.

[2] Kuroda, S. T., Stationary theory of scattering and eigenfunction expansions, I, II, Sûgaku, 18 (1966), 74-85; 137-144. (Japanese)

[3] - An abstract stationary approach to perturbation of continuous spectra and scattering theory, J. Analyse Math. 20 (1967), 57-117.

[4] - Construction of eigenfunction expansions by the perturbation method and its application to $n$-dimensional Schrödinger operators, MRC Technical Summary Report $\$ 744$, Univ. Wisconsion, 1967.

[5] Konno, R. and S. T. Kuroda, On the finiteness of perturbed eigenvalues, J. Fac. Sci. Univ. Tokyo, Sect. I, 13 (1966), 55-63.

[6] Sobolev, S. L., On a theorem of functional analysis, Mat. Sb. 4 (1938), 471-497. (Russian)

[7] , Applications of functional analysis, in mathematical physics, A. M. S. Transl. Math. Monogr. vol. 7, 1963.

[8] Ikebe, T. and T. Tayoshi, Wave and scattering operators for second order elliptic operators in $R^{3}$, Publ. RIMS Kyoto Univ. Ser. A, 4 (1968), 483-496. 
